achylia, nervous vomiting, hyperesthesia, gastralgia, intestinal neuroses, nervous diarrheas, enteralgia), as well as in acute and chronic catarrh of the stomach and bowel, simple peptic and intestinal ulcer, parasites, etc.

We must recognize the limitations as well as the value of this remarkable method of examination.

In closing, I wish to thank Dr. Ariel W. George, of Boston, for the opportunity to examine and discuss many excellent $x$-ray plates.

\section{THE EXPERIMENTAL PRODUCTION OF LESIONS RESEMBLING PELLAGRA.*}

BY HERMAN M. ADLER, M.D., BOSTON,

IN presenting this account of certain observations that were made in the course of experiments conducted during the past three years, it should be understood that it is not my intention to enter the lists for or against any of the present theories of the causation of pellagra. The small number of clinical cases of pellagra that we in the North are given the opportunity of seeing preclude our arriving at any very complete conception of this disease. On the other hand, the very fact that cases do occur in the North under conditions which at first sight seem radically different from those which are believed to be predisposing to the occurrence of pellagra should lead us to consider that this disease may be of more general and fundamental importance than is usually conceded.

In the course of the past two years, without any special effort on my part, four cases of pellagra have come to my notice, all inmates of Massachusetts institutions. ${ }^{1}$ Undoubtedly, as the interest in this disease is further aroused, more cases will come to our attention. It is clear, then, that pellagra is not without considerable importance for us in the North, as has been pointed out by many authors, and it becomes necessary to inquire into its significance. The question that immediately presents itself is perhaps comparable to the question that is raised by the occurrence of such diseases as pernicious anemia, the results of arteriosclerosis, et cetera, in the insane.

In all diseases we are accustomed to consider two main factors: the one, the causative factor; the other, the reaction of the individual to the causative agent. The importance that has been given to the infectious diseases on account of the remarkable advances in this branch of knowledge during the past decades has, perhaps, diverted the attention of most investigators to a more or less degree from the consideration of the second factor, that of the reaction of the individual to the causative agent. When, however, we undertake the investigation of the chronic diseases, of which arteriosclerosis and various forms of insanity are perhaps the most

* From the Laboratory of the Danvers State Hospital. No. 25 of the Danvers State Hospital Series. striking examples, the importance of the particular causative agent in any individual case is often overshadowed by that of the reaction of the individual. In a case of arteriosclerosis, for instance, it is not enough to discover that the pneumococcus has infected the lungs and is causing pneumonia, but the all-important point is, what is the individual's reaction to this organism. In one case we may find that the result is a typical, straightforward lobar pneumonia ; in another case we may find practically none of the symptoms of lobar pneumonia, but indefinite symptoms and signs of a low-grade septic infection running a more or less rapidly fatal course with or without mental symptoms, and offering at no time a clinical picture which can in any sense be called specific for the pneumococeus. It is this point of view which is essential in considering the mechanism of most chronic diseases, and, in my opinion, it is this point of view which is of importance in attempting to determine the clinical significance of pellagra.

In the course of an investigation conducted with the object of determining the reasons for emaciation and anemia in many chronic diseases found among the insane and senile, the following main points have been made. ${ }^{2}$. It has been found that certain cases of emaciation were suffering from the effects of a fat intoxication. This is a condition which has long been the object of attack by pediatricians. While in the realm of infant diseases the main stress has been laid on the intoxications with foreign proteins, there are a number of instances on record where loss of weight, gastrointestinal disturbances and anemia, often approaching the pernicious type, have been observed as the direct effect of intoxication by foreign fat.

A similar condition has been shown to exist in certain cases of extreme emaciation due to general paralysis. ${ }^{3}$. In these cases it was possible to demonstrate an autohemolysine in the serum after overfeeding with fat in the form of milk or cream. The autohemolysines disappeared regularly when the fats were reduced. These experiments pointed to the existence of a defect in the ability of the individual for handling what under normal conditions was a food. It is an instance of an intoxication by a substance which ordinarily should help rather than harm the organism. The work of Faust and Tallcuist ${ }^{4}$ leads us to assume that the hemolysine in this case was oleic acid or some similar chemical substance.

Experiments on the production of pernicious anemia in rabbits by overfeeding with olive oil, cotton-seed oil, chemically pure triolein, etc., have brought out the facts that an excess of fat may be absorbed by the intestines; that, having been absorbed, it will produce at times the symptoms of acute enteritis, loss of weight and in time a varying degree of anemia, which may be severe enough to closely resemble the pernicious type. In these experiments ten rabbits were subjected to chronic poisoning with oil. Of 
these, three had previously been fed daily for about a year with 0.3 gram of quinine. Three rabbits had been treated for three months with intravenous injection of 0.01 gram of quinine daily. The remaining four received daily feedings of olive oil without previous treatment. Five cubic centimeters per kilo weight of olive oil was the dose given to all of the rabbits." All of these rabbits developed the blood picture of a secondary anemia within a few days. In four the blood picture of pernicious anemia developed in from two to three months and lasted for a few days. The weight curve followed approximately the appearances in the blood, dropping sharply as the anemia became marked, rising as the anemia improved. The weight and anemia improved markedly whenever the feeding of oil was temporarily suspended.

In six out of these ten rabbits there appeared during the latter part of the experiment, four months or more after oil feeding was commenced, a skin eruption on the inner surface of the ears. This condition was in the nature of a diffuse lesion, characterized by intense congestion and thickening of the entire ear. The ears would become so much thickened that they could be folded over with difficulty, and the congestion caused them at times to appear dark purplish in color. This appearance of the ears was invariably accompanied by acute enteric symptoms, profuse diarrheas and bloody stools. At the same time the weight of the animal dropped rapidly. The oil feedings were discontinued and the symptoms lessened in severity and disappeared. The ears gradually improved until little remained except a few crusts. On feeding the oil again, frequently the intestinal symptoms returned, but only in one case has the eruption in the ears increased in severity a second time. Five out of the six rabbits showed the ear lesion during the months of June, July and August, improving through the fall. One animal showed the ear lesion in May and improved in the course of the summer. The three rabbits that had been fed quinine for a year previous to the oil feedings at no time showed any signs of an eruption or inflammation in the ears. The fourth rabbit that failed to show these symptoms was one of the three that had been treated with the intravenous injection of quinine.

During the entire experiment the animals were kept in all other respects under the same conditions as they had been previously, and under the same conditions and in part in the same cages with control rabbits that were not under experimentation. Not a single rabbit in our entire animal house at Danvers, out of some 25 to 30 animals, showed anything resembling this eruption, except the rabbits mentioned. The rabbits were not kept isolated in any sense, and all were fed alike, the same food, in the same

* This dose was determined upon after experiment in which it was found that a dose of 10 c.c. of oil per kilo of bodv weight produced experimentally violent diarrheas, and rapid loss of weight, kilo of body weight suffices to kill occasionally, two or three doses invariably. amounts, from the same stock. The only difference between the animals under experimentation and those not, lay, so far as could be ascertained, in the feedings of oil.

It would seem that there were several factors involved in the production of this condition. In the first place, we have the introduction of a large amount of oil, the chief constituent of which is triolein. This triolein is split up in the intestine, in part at least, into glycerine and oleic acid, and the oleic acid is absorbed. Oleic acid is a hemolytic agent, and, unless neutralized in some fashion, will cause destruction of red cells. It appeared, furthermore, from experiments conducted upon the brains of cats ${ }^{5}$ that oleic acid is a neurolytic as well as a hemolytic agent, that is, it will destroy by lysis the nerve cell in the same way that it destroys the red blood cell. It is very probable that this lytic action of oleic acid is not confined to these two types of cell.

In the second place, we have to consider here the effect upon the intestine. This seems to be due to the irritating action of the hemolytic oleic acid, probably not only upon the red cell but also upon the smooth muscle coats of the intestine. At autopsy an intestine in this condition shows a marked congestion of the mucosa and submucosa, especially in the region of the lymphoid cells beneath the epithelium of the villi. The cathartic action of olive oil has long been known to the clinician. Its absolute harmlessness in all doses, however, may be doubted in view of these findings.

In the third place, we have to consider the production of a superficial eruption on the skin in one part of the rabbit, namely, the ears. The only immediate reason that I can assign for this site is that it is the only place on the body of a rabbit which is not covered by fur. Attempts to prove this point by shaving parts of the body, failed on account of the length of time required for the reaction and the practical difficulties of keeping the animal shaved closely for such a period. This part of the work is at present being repeated. There were at no time in any of these animals any symptoms pointing to an involvement of the central nervous system, nor were any lesions discovered post mortem.

These experiments seem to be presumptive evidence that a condition which is characterized by a dermatitis and acute enteritis need not be necessarily a clinical entity in the sense of being a specific reaction to a specific causative agent, but that this may be a more or less general reaction common to a large number of pathological conditions. If we assume that the mechanism of this sort of disease includes on the one hand a causative agent of the general nature of the chemical used in these experiments, on the other hand a protective apparatus which has a greater or less degree of strength, we can conceive the production of the same symptoms as a result of considerable variation in the exact mechanism. 
In the first place, the causative agent may be so powerful as to overwhelm the protective apparatus of the best equipped individual. This powerful effect need not depend upon quantity. For instance, it is accepted that the hemolytic action of oleic acid depends on the fact that it is unsaturated. Oleic acid, however, contains only one double bond or unsaturated bond in its molecule. There are substances of the general chemical and physical nature of oleic acid which are unsaturated, which contain two or more double bonds in the molecule and which are, therefore, more toxic, more hemolytic, so that the dose required of such a substance may be actually very much smaller than that of oleic acid and still act pharmacologically more powerfully. It is well known that a great variety of such unsaturated fats or fatty acids occur in nature. Such unsaturated fatty acids, which in general are classed with the drying oils or the semi-drying oils, are frequently constituents of our foods, as Mizell ${ }^{6}$ and others have pointed out. This is true of the North as well as of the South, where the butter substitutes are in use in considerable quantities. These facts do not, however, preclude the possibility of the introduction or production of such a substance by means of parasites or bacteria, and thus the reaction would be rather to a chemical substance regardless of where it came from, than to the particular living thing that produced the fat.

On the other hand, we have the other factor of the defense of the organism. It has been shown by Stheeman ${ }^{7}$ that the chief defensive apparatus for toxic fats is the lymphoid tissue. This lymphoid tissue is found in the body arranged in the lymph glands and spleen, and distributed through the various organs to a greater or less degree, notably in the submucosa of the intestine. Now, it has been observed that the lymphoid tissue of certain cases of insanity and the lymphoid tissue in senescence is diminished quantitatively. The experiments quoted above, in which hemolytic substances were introduced into the blood stream by overfeeding with fat, were carried out successfully only in cases in which the emaciation suggested a partial atrophy of the lymphoid tissue:

There is one point which is, perhaps, of the most striking importance in connection with this disease, namely, the relation of sunlight to the production of the eruption. While my experiments have not yet progressed to the point where I can speak with any degree of certainty upon this matter, I am inclined to the belief that sunlight is merely the exciting irritant, but has nothing to do with producing the condition of irritability in the skin. Instead of an ordinary sunburn, the individual is injured to the extent of inflammation, just as some fair-haired people with little pigment in their skin may suffer from an acute inflammation as a result of a slight exposure to the summer sun, which in a brunette would cause little inconvenience. Just what is the mechanism of the production of this state o irritability remains still to be determined. There is, however, considerable evidence in support of the conception that the same condition which produces congestion and peristalsis in the intestine will predispose the skin to harmful attack by light. I hope that in the near future I may be able to report further on this phase of the problem.

\section{REFERENCES.}

1 White, D.: A Case of Pellagra in New England. J. A. M. A., 1912, vol. lviii, p. 1279. The other three cases (unpublished) seen at Danvers State Hospital and Boston State Hospital.

2 Adler, H. M. : Proceedings of the Society for Experimental Biology and Medicine. October, 1911.

${ }^{8}$ Adler, H. M.: Some Effects of Overfeeding Fats in Certain Cases of Insanity. BOston MEd. AND SURG. Jour., vol. clxiii, No. 5, p. $225,1910$.

4 Faust and Tallquist: Archiv. f. exp. Path. u. Pharm., vol. lvii, p. 375,1907 .

5 Adler, H. M.: Proceedings of the American Society for the Ad. vancement of Clinical Investigation. April, 1912.

- Mizell, G. C.: Etiology, Pathology and Treatment of Pellagra. Atlanta Jour.-Rec. Med., 1911, vol. lviii, pp. 401, 523.

7 Stheeman, H. A.: Beiträge zur Patholog. Anat. u. z. allgemeine Patholog., vol. xlvii, p. $170,1910$.

\section{THE PRESENT POSITION OF ABDOMI- NAL CESAREAN SECTION IN ECLAMP. SIA.*}

BX JOHN T. WILLIAMS, M.D., BOstON.

THIs article represents the result of an unprejudiced inquiry into the actual value of Cesarean section in eclampsia as shown by the reported cases in which it has been performed. In spite of the recent wave of conservatism in the treatment of eclampsia which has swept over the obstetrical world, there are still many who hold that immediate delivery is the first essential in successfully coping with this condition.

For rapid delivery in eclampsia there are three methods from which to choose:

1. Manual or instrumental dilatation of the cervix.

2. Vaginal Cesarean section.

3. Abdominal Cesarean section.

The chief obstacle to the delivery of eclamptics is the boardlike rigidity of the undilated cervix, which seems to be especially marked in eclamptics; and although the majority of the patients are primigravidae, it often occurs in those who are multigravidae as well.

The dilatation of such a cervix is difficult, tedious and exhausting, and accompanied by considerable shock, and the danger of serious laceration of the cervix and lower segment of the uterus.

To overcome these disadvantages, Dührssen devised the operation of vaginal Cesarean section. The technical difficulties of this operation are considerable. Its performance requires a considerable degree of skill and experience in vaginal operating, at least two assistants, and good illumination; conditions difficult to obtain except in a hospital.

* Part of a paper read by invitation before the Everett Medical Society, May 8, 1912 